LM-03K053

June 13, 2003

\title{
Measurement of the Auger Recombination Rate in p-type 0.54-eV GalnAsSb by Time-Resolved Photoluminescence
}

S. Anikeev, D. Donetsky, G. Belenky, S. Luryi, C.A. Wang, J.M. Borrego, G. Nichols

\footnotetext{
This report was prepared as an account of work sponsored by the United States Government. Neither the United States, nor the United States Department of Energy, nor any of their employees, nor any of their contractors, subcontractors, or their employees, makes any warranty, express or implied, or assumes any legal liability or responsibility for the accuracy, completeness or usefulness of any information, apparatus, product or process disclosed, or represents that its use would not infringe privately owned rights.
} 


\title{
Measurement of the Auger recombination rate in p-type
}

\subsection{4-eV GaInAsSb by time-resolved photoluminescence}

S. Anikeev, D. Donetsky, G. Belenky, and S. Luryi

State University of New York, Stony Brook, NY 11794-2350

C. A. Wang

Lincoln Laboratory, Massachusetts Institute of Technology, Lexington, MA 02420-9108

J. M. Borrego

Rensselaer Polytechnic Institute, Troy, NY 12180

G. Nichols

Lockheed Martin Corporation, Schenectady, NY 12301

\begin{abstract}
Auger recombination in $p$-type GaSb, InAs and their alloys is enhanced due to the proximity of the bandgap energy and the energy separation to the spin split-off valence band. This can affect the device performance even at moderate doping concentration. We report electron lifetime measurements in a p-type 0.54-eV GaInAsSb alloy, commonly used in a variety of infrared devices. We have studied a series of double-capped heterostructures with varied thicknesses and doping levels, grown by organometallic vapor phase epitaxy on $\mathrm{GaSb}$ substrates. The Auger coefficient value of $2.3 \times 10^{-28} \mathrm{~cm}^{6} / \mathrm{s}$ is determined by analyzing the photoluminescence decay constants with a systematic separation of different recombination mechanisms.
\end{abstract}


The GaInAsSb quaternary alloy lattice matched to GaSb is an attractive material for mid-infrared (IR) optoelectronic devices, and is considered to be one of the primary materials for thermophotovoltaic (TPV) energy conversion systems that operate with thermal sources heated to approximately $1000{ }^{\circ} \mathrm{C} .{ }^{1-3}$ To maximize overall TPV system efficiency, the band-gap energy $E_{g}$ of the absorber (emitter layer) should match the peak emission wavelength of the infrared radiator. Thus, GaInAsSb alloy compositions corresponding to $\mathrm{E}_{\mathrm{g}}=0.5-0.6 \mathrm{eV}$ are of particular interest. TPV devices utilize a $p$-type emitter to take advantage of higher minority electron mobility and therefore longer diffusion length. $0.52-0.55-\mathrm{eV}$ GaInAsSb TPV cells fabricated by organometallic vapor phase epitaxy (OMVPE) demonstrate high performance. The internal quantum efficiency of $p$-on- $n$ devices with a 4- $\mu$ m-thick emitter doped to $2 \times 10^{17} \mathrm{~cm}^{-3}$ was reported to approach the theoretical limit of $100 \% .{ }^{3}$ However, the open circuit voltage $V_{o c}$ was only $330 \mathrm{meV}$, which is about $60 \%$ of the material $\mathrm{E}_{\mathrm{g}}$. This indicated the need for further improvement.

Optimization of the GaInAsSb emitter doping level is a critical issue for TPV cell design. On the one hand, higher dopant concentration boosts the diode built-in potential, which is beneficial for increasing the open-circuit voltage. On the other hand, conversion efficiency degrades with higher doping due to radiative and Auger recombination losses. The effect of radiative recombination on the device performance is alleviated by reabsorption of the emitted photons, known as the photon recycling effect. Therefore in TPV diodes with higher emitter doping levels, Auger recombination can be regarded as a major mechanism limiting device performance. In this paper, recombination processes in 
GaInAsSb were studied for p-doping concentration up to $2 \times 10^{17} \mathrm{~cm}^{-3}$, which is a typical level for TPV devices ${ }^{2,3}$.

In III-V compound materials, such as GaSb, InAs and a number of their alloys, the magnitude of $E_{g}$ is similar in value to the spin-orbit splitting energy. ${ }^{4-5}$ In this case, the Auger process that generates holes in the split-off valence band (CHHS) occurs with small momentum transfer and, therefore, low activation energy. This leads to an enhanced rate of Auger recombination, as was first suggested by Takeshima ${ }^{6}$ and experimentally shown in the pioneering work by Benz and Conradt. ${ }^{7}$

Auger recombination has been extensively studied in bulk and epitaxial GaSb, InAs and some GaSb-based ternary alloys. ${ }^{8-9}$ In contrast, there have been only a few reported measurements of Auger lifetimes in epitaxial GaInAsSb. Interpolation of the limited data reported in the literature for III-V compounds yields spreading of Auger coefficient for $0.54-\mathrm{eV}$ materials within two orders of magnitude. ${ }^{\prime}$ Auger lifetimes in p$\mathrm{Ga}_{0.84} \mathrm{In}_{0.16} \mathrm{As}_{0.14} \mathrm{Sb}_{0.86}$ layers grown by liquid phase epitaxy were determined by Riech et al. ${ }^{10}$ The authors applied a photo-acoustic (PA) technique, which allowed monitoring the heat generated in the sample. Lifetime values were indirectly obtained by fitting the experimentally measured PA phase signal, a procedure that required prior knowledge of a number of thermal and recombination parameters of the material.

In this letter, we report the lifetime in p-type 0.54-eV GaInAsSb determined by a direct method from the transient photoluminescence (PL). Time-resolved PL is an alternative to radio-frequency photoconductive decay method used in the mid-IR wavelength range for lifetimes greater than 10-20 ns. ${ }^{11-12}$ The setup we developed allows measurement of the luminescence decay in the mid-IR range by a $\mathrm{HgCdTe}$ mesa 
photodiode. The overall time resolution of the detection system is better than $5 \mathrm{~ns}$. Details of the setup were described earlier. ${ }^{16}$

Three sets of $\mathrm{GaSb} / \mathrm{p}-\mathrm{Ga}_{0.85} \mathrm{In}_{0.15} \mathrm{As}_{0.14} \mathrm{Sb}_{0.86} / \mathrm{GaSb}$ double heterostructures (DHs) with active layers $\mathrm{Zn}$-doped to $1 \times 10^{16}, 1 \times 10^{17}$, and $2 \times 10^{17} \mathrm{~cm}^{-3}$ were grown by OMVPE on (100) $\mathrm{n}-\mathrm{GaSb}$ substrates misoriented $6^{\circ}$ toward (111)B. The GaInAsSb layer thickness $(W)$ was varied from 1.2 to $5 \mu \mathrm{m}$. GaSb confinement layers (caps) were 50-nm-thick and nominally undoped but known to be lightly $p$-type, with $p=1 \times 10^{16} \mathrm{~cm}^{-3}$. The 4-K PL spectra exhibited a full width at half maximum of 5 to $12 \mathrm{meV}$, with the smallest values being measured for the most lightly doped samples. Absorption in the front $\mathrm{GaSb}$ cap layer was estimated to be $12 \%$ of the incident excitation power. The 300-K PL spectra demonstrated a single peak at $2.3 \mu \mathrm{m}$ exhibiting no evidence of luminescence from the cap.

Figure 1 shows the typical PL decay for DHs with different thicknesses. The transient response was measured under low excitation conditions. Under these conditions, the decay time $\tau_{\mathrm{PL}}$ is a characteristic of the structure, and independent of the concentration of photogenerated electrons. Non-equilibrium electron-hole pairs are generated in the active region near the interface. Quasi-equilibrium electron distribution across the active layer is established by diffusion within the time $\tau \sim W^{2} / D$, where $D$ is the diffusion coefficient. For the thickest samples with $W=5 \mu \mathrm{m}$ and minority electron mobility of $5 \times 10^{3} \mathrm{~cm}^{2} / \mathrm{s}^{13}$, the diffusion time $\tau$ is about $2 \mathrm{~ns}$.

Under conditions of low excitation $\Delta n<<p$ and low interfacial recombination velocity $S<<D / W$, the bulk and the interface recombination processes can be separated as follows: 


$$
I / \tau_{P L}=1 / \tau_{N R}+B p / \phi(W)+2 S / W
$$

The inverse bulk lifetime is described by the first two terms in (1) where $\tau_{N R}$ is the nonradiative lifetime and $B$ is the radiative recombination coefficient. The radiative term is thickness-dependent due to the effect of reabsorption, as given by Asbeck's photon recycling factor $\phi(W) .{ }^{14}$ The factor $\phi$ monotonically increases with the active layer thickness, and hence in the limit of infinite thickness the PL decay constant approaches the non-radiative bulk lifetime. The photon recycling effect results in a deviation from the linear relation between $I / \tau_{P L}$ and $I / W$. This non-linearity is well pronounced in structures with very small $S$ when radiative recombination dominates over interfacial recombination. ${ }^{15}$ Our experiments correspond to the opposite limit of large interfacial recombination, where $I / \tau_{P L}$ increases with $I / W$ linearly with a slope that is governed mostly by $S$ and an offset that determines the inverse non-radiative bulk lifetime.

To estimate the contribution of radiative recombination, $\phi$ was calculated using the approach developed in Ref. 14 and is shown in Figure 2. The calculation was based on the measured absorption spectrum (inset in Figure 2). Assuming $B=5 \times 10^{-11} \mathrm{~cm}^{3} / \mathrm{s}^{12}$ in the most critical case of $p=2 \times 10^{17} \mathrm{~cm}^{-3}$, the radiative lifetime ranges from approximately 0.3 to $0.7 \mu$ s for $W$ varied from 1 to $5 \mu \mathrm{m}$. The measured inverse PL decay time $1 / \tau_{P L}$ versus inverse active layer thickness $(1 / W)$ is shown in Figure 3, and demonstrates linear behavior (dashed lines) for all three sets of DHs. The interfacial recombination velocity that is extracted from the slope is slightly overestimated due to the contribution of radiative recombination. This contribution was subtracted from the linear fit and the results shown by solid lines reflect the actual interfacial recombination 
velocity. Even in structures with the highest doping $\left(p=2 \times 10^{17} \mathrm{~cm}^{-3}\right)$ the relative error in $S$ does not exceed $5 \%$.

The slope of the $1 / \tau_{P L}$ versus $1 / W$ plots was found to be quite sensitive to the doping level in the GaSb caps. This effect, considered in our previous work, was explained by accumulation of minority carriers at the GaSb/GaInAsSb heterointerface ${ }^{16}$ and experimentally confirmed the dominant role of interfacial recombination.

Values of non-radiative bulk lifetime $\tau_{N R}$ were determined from the y-intercepts in Figure 3. Table I summarizes the obtained recombination parameters for DHs with different doping concentrations. The nonradiative bulk lifetimes are made up of contributions from Shockley-Read-Hall (SRH) and Auger recombination processes. Under low excitation, SRH recombination in $p$-type materials proceeds via capture of minority electrons by deep (midgap) levels. In this commonly used approximation, the SRH lifetime is determined by the defect density and does not depend on the doping level. ${ }^{17}$ Consequently, the doping dependence of $\tau_{N R}$ is given by

$$
1 / \tau_{N R}=1 / \tau_{S R H}+C_{p} p^{2}
$$

where $C_{p}$ is the Auger recombination coefficient. Figure 4 plots $1 / \tau_{N R}$ versus the square of the acceptor concentration in the active region. Using a linear fit, both the Auger coefficient and the SRH lifetime were deduced, resulting in the values of $C_{p}=2.3 \times 10^{-28}$ $\mathrm{cm}^{6} / \mathrm{s}$ and $\tau_{S R H}=1.3 \mu \mathrm{s}$, respectively. Since the PL decay constants are determined with accuracy better than $\pm 1 \mathrm{~ns}$, the error in $C_{p}$ value is mostly controlled by the knowledge of 
the doping concentration and reproducibility of growth. The standard error in $C_{p}$ is estimated to be $\pm 0.8 \times 10^{-28} \mathrm{~cm}^{6} / \mathrm{s}$.

In summary, minority carrier lifetimes were measured directly by the study of the photoluminescence kinetics in p-type 0.54-eV GaInAsSb alloys for doping concentrations of up to $2 \times 10^{17} \mathrm{~cm}^{-3}$, as typically used in TPV devices. Different recombination mechanisms were separated using the dependence of lifetime on the structure thickness and the doping concentration. 


\section{Figure Captions}

Figure 1. Normalized photoluminescence decay in p-GaInAsSb double heterostructures doped at $1 \times 10^{17} \mathrm{~cm}^{-3}$ for the active layer thickness of 1.5 and $4 \mu \mathrm{m}$.

Figure 2. Inverse photon recycling factor versus inverse GaInAsSb layer thickness. The measured fundamental absorption edge of the active layer is shown in the inset.

Figure 3. Inverse photoluminescence decay time versus inverse structure thickness for three sets of p-GaInAsSb double heterostructures doped at $2 \times 10^{17}, 1 \times 10^{17}$, and $1 \times 10^{16}$ $\mathrm{cm}^{-3}$. The dashed lines show a linear fit of the experimental data. The solid lines reflect the actual interfacial recombination velocity (the radiative recombination excluded).

Figure 4. Inverse nonradiative bulk lifetime versus square of the acceptor concentration in GaInAsSb epilayers. 


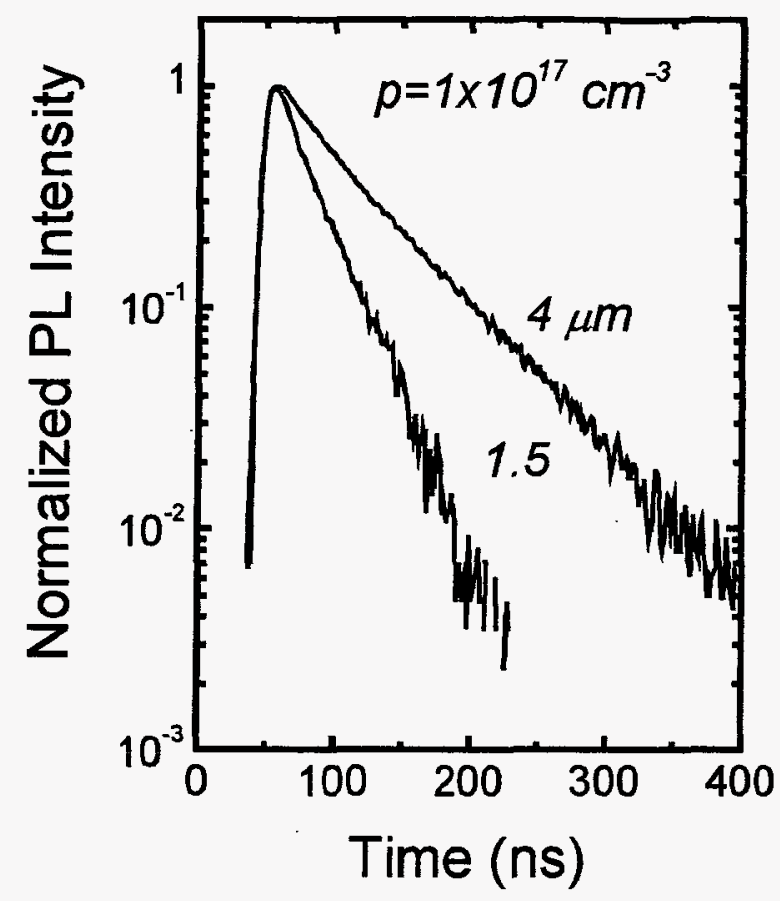

Figure 1. 


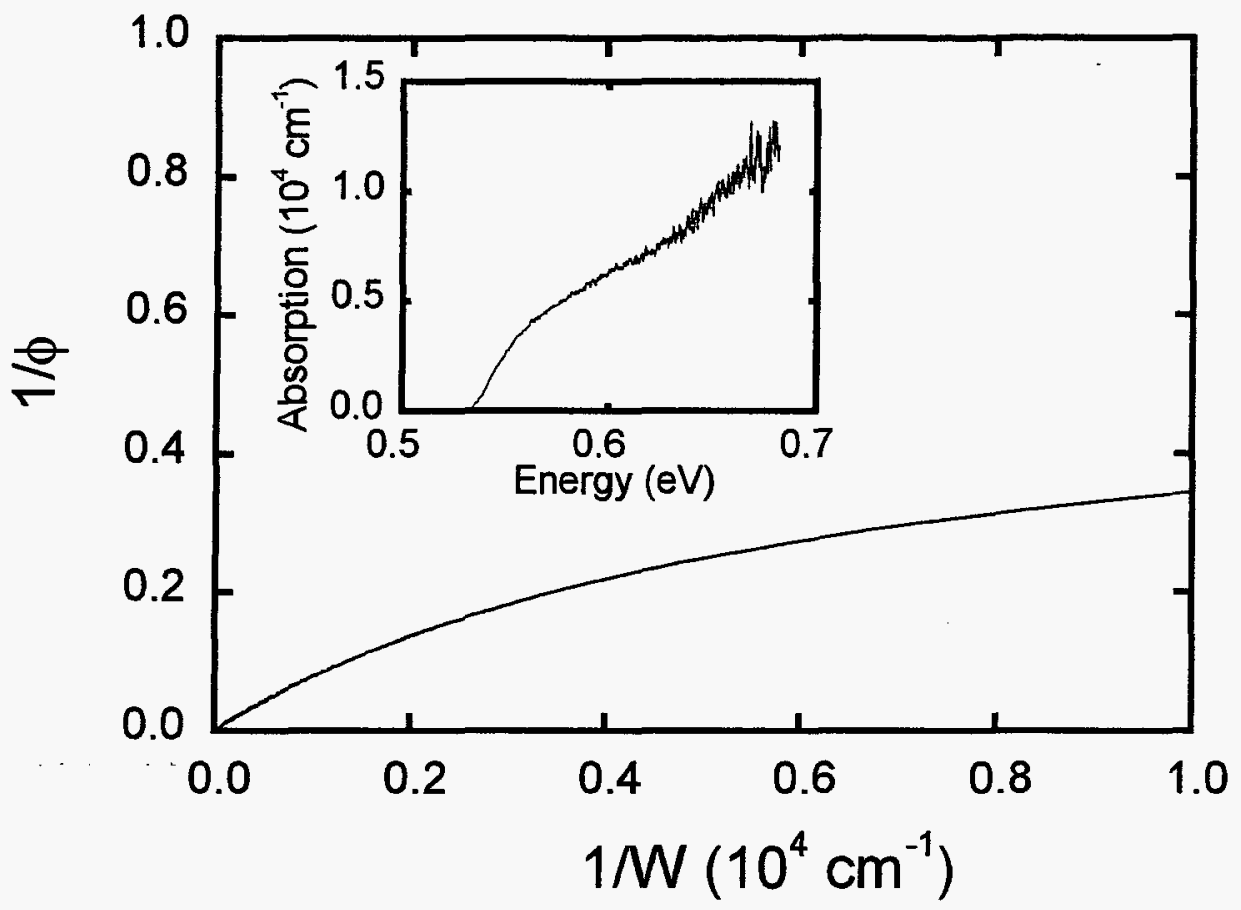

Figure 2.

10 


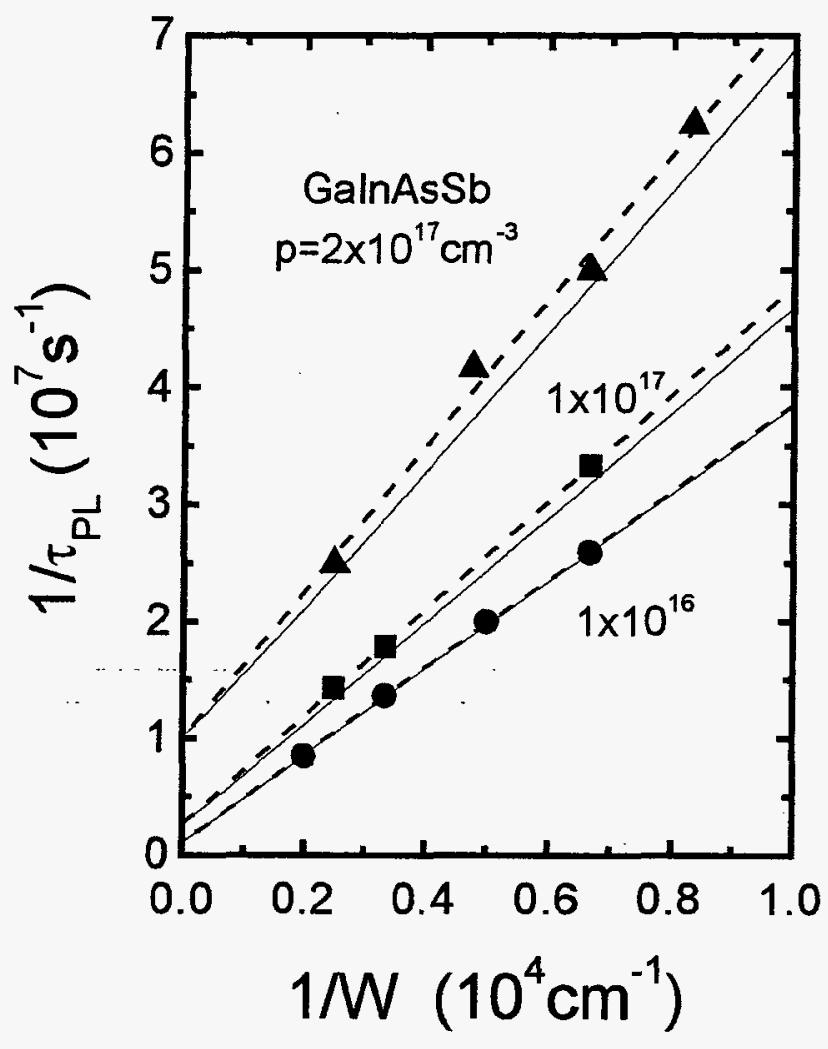

Figure 3. 


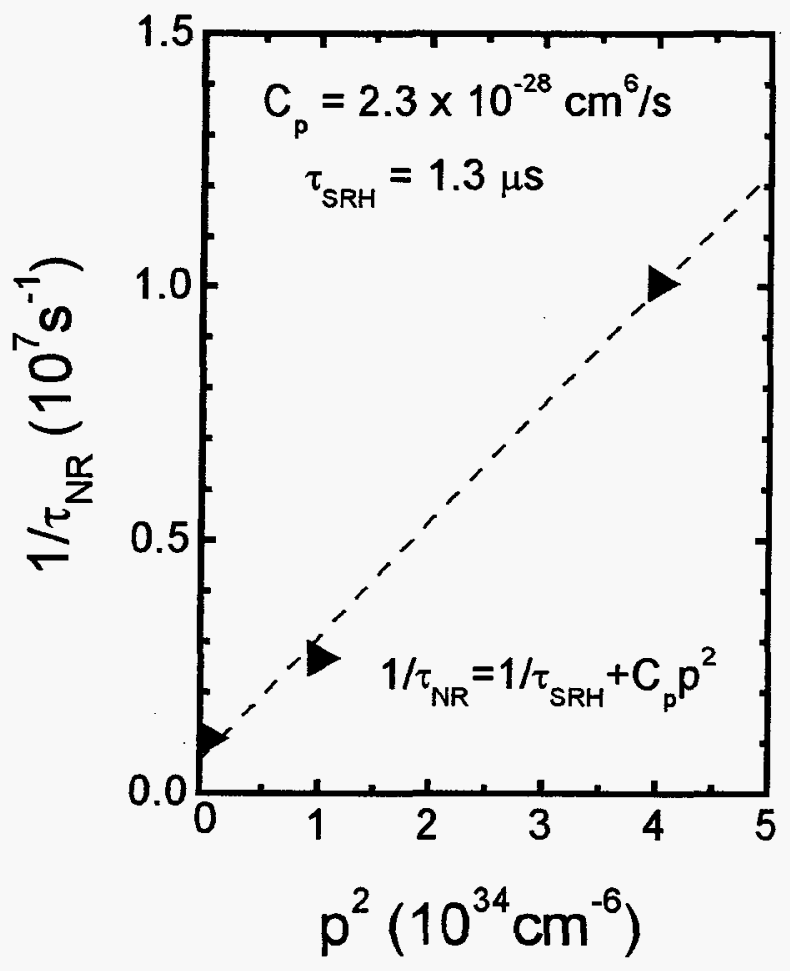

Figure 4. 


\begin{tabular}{|c|c|c|}
\hline $\begin{array}{c}\text { GaInAsSb Doping } \\
\text { Concentration } \\
\mathrm{p}\left(\mathrm{cm}^{-3}\right)\end{array}$ & $\begin{array}{c}\text { Interfacial Recombination } \\
\text { Velocity } S(\mathrm{~cm} / \mathrm{s})\end{array}$ & $\begin{array}{c}\text { Nonradiative Bulk Lifetime } \\
\text { (ns) }\end{array}$ \\
\hline $2 \times 10^{17}$ & 3100 & 100 \\
\hline $1 \times 10^{17}$ & & \\
& & \\
\hline $1 \times 10^{16}$ & 2300 & 370 \\
\hline
\end{tabular}

Table 1. Recombination parameters for GaInAsSb/GaSb DHs. 


\section{References}

${ }^{1}$ G. W. Charache, P. F. Baldasaro, L. R. Danielson, D. M. DePoy, J. Freeman, C. A. Wang, H. K. Choi, D. Z. Garbuzov, R.U. Martinelli, V. Khalfin, S. Saroop, J.M. Borrego, and R. J. Gutmann, J. Appl. Phys., 85, 2247 (1999).

${ }^{2}$ H. K. Choi, C. A. Wang, G. W. Turner, M. J. Manfra, D. L Spears, G. W. Charache, L. R. Danielson, D. M. DePoy, Appl. Phys. Lett., 71, 3758 (1997).

${ }^{3}$ C.A. Wang, C.J. Vineis, H.K. Choi, M.K. Connors, R.K. Huang, L.R. Danielson, G. Nichols, G.W. Charache, D. Donetsky, S. Anikeev, and G. Belenky, AIP Conf. Proc., 653, 324 (2003).

${ }^{4}$ I. Vurgaftman, J. R. Meyer, L. R. Ram-Mohan, J. Appl. Phys., 89, 5815 (2001).

${ }^{5}$ M. Munoz, K. Wei, Fred H. Pollak, J. L. Freeouf, C. A. Wang, and G. W. Charache, J. Appl. Phys., 87, 1780 (2000).

${ }^{6}$ M. Takeshima, J. Appl. Phys., 43, 4114 (1972)

${ }^{7}$ G. Benz and R. Conradt, Phys. Rev. B, 16, 843 (1977) 
${ }^{8}$ A. N. Titkov, G. N. Iluridze, I. F. Mironov, V. A. Cheban, Sov. Phys. Semicond., 20, 14 (1986)

${ }^{9}$ G. N. Tluridze, I. F. Mironov, A. N. Titkov, V. A. Cheban, Sov. Phys. Semicond., 20, $310(1986)$

${ }^{10}$ I. Riech, M. L. Gomez-Herrera, P. Diaz , J. G. Mendoza-Alvarez, J. L. Herrera-Perez, and E. Marin, Appl. Phys. Lett., 79, 964 (2001).

${ }^{11}$ R. K. Ahrenkiel, AIP Conf. Proc., 353, 161 (1996).

${ }^{12}$ J. M. Borrego, S. Saroop, R. J. Gutmann, G. W. Charache, T. Donovan, P. F. Baldasaro, and C. A. Wang, J. Appl. Phys., 89, 3753 (2001).

${ }^{13}$ C. A. Wang, J. Crystal Growth, 191, 631 (1998).

${ }^{14}$ P.Asbeck, J. Appl. Phys., 48, 820 (1977).

${ }^{15}$ R. K. Ahrenkiel, B. M. Keyes, G. B. Lush, M. R. Melloch, M. S. Lundstrom, H. F. MacMillan, J. Vac. Sci. Technol., A 10, 990 (1992).

${ }^{16}$ D. Donetsky, S. Anikeev, G. Belenky, S. Luryi, C. A. Wang, and G. Nichols, Appl. Phys. Lett., 81, 4769 (2002). 
${ }^{17}$ R. K. Ahrenkiel, in Semiconductors and Semimetals 39, R. K. Ahrenkiel and

M. S. Lundstrom, Eds. (Academic Press, New York; 1993) pp. 39-150. 